\title{
ON A METHOD OF PROOF FOR THE MINIMAX THEOREM ${ }^{1}$
}

\section{HUKUKANE NIKAIDÔ}

In this brief note the minimax theorem on convex games played on (possibly infinite-dimensional) convex sets will be proved in a new elementary way. Although the theorem is not new, the method of proof seems to be new. The proof will be done along the customary line of dealing with minimization problems by means of calculus. Neither the separation theorem on convex sets nor any fixed-point techniques will be needed. The result was obtained by a closer study of the Brown-von Neumann's differential equation solving games with skew-symmetric payoff matrices [1]. The method will also be applied to prove the duality in linear programming.

1. The minimax theorem. Let $X$ and $Y$ be convex sets which are compact with respect to certain given Hausdorff topologies. It is also assumed that for any fixed $x, u \in X, y, v \in Y$, the mappings $t \rightarrow(1-t) x+t u:[0,1] \rightarrow X$ and $t \rightarrow(1-t) y+t v:[0,1] \rightarrow Y$ are continuous in these topologies. Let further be given a real-valued function $f(x, y)$ on the product set $X \times Y$ that fulfills the following conditions:

(1) For any fixed $y \in Y, f(x, y)$ is a continuous concave function of $x$ on $X$.

(2) For any fixed $x \in X, f(x, y)$ is a continuous convex function of $y$ on $Y$.

Under these assumptions the minimax theorem asserts: There is at least one saddle point $(\hat{x}, \hat{y}) \in X \times Y$ such that $f(x, \hat{y}) \leqq f(\hat{x}, \hat{y}) \leqq f(\hat{x}, y)$ for all $(x, y) \in X \times Y$.

The standard methods of proving this theorem so far known are more or less based on separation theorems on convex sets, and there are numerous proofs along this line. Another method was given in $[3 ; 4 ; 6]$ to prove the theorem under more general conditions by making use of Brouwer's fixed-point theorem. It is also noted that the wellknown method based on Kakutani-type fixed-point theorems can not directly be applied to the theorem stated above, since $f(x, y)$ is not necessarily continuous with respect to $(x, y)$.

2. Proof of the theorem. The proof begins with skew-symmetrizing the payoff in a familiar fashion. Put $K((u, v),(x, y))=f(u, y)-f(x, v)$, $(u, v),(x, y) \in X \times Y$. It then satisfies:

Received by the editors July 6, 1958.

1 An earlier version of this note was published in Japanese [5]. 
(3) For any fixed $(u, v) \in X \times Y, K((u, v),(x, y))$ is a continuous convex function of $(x, y)$ on $X \times Y$ in the cartesian product topology. This topology certainly makes $X \times Y$ a compact convex set so that the convex combination of any two fixed points depends continuously on the coefficient $t$. Further, $K((u, v),(x, y))$ is skew-symmetric in the sense that

$$
K((u, v),(x, y))+K((x, y),(u, v))=0 .
$$

As is well-known and easily seen $[1 ; 2 ; 4],(\hat{x}, \hat{y})$ is a saddle point for $f(x, y)$ on $X \times Y$, if and only if

$$
K((u, v),(\hat{x}, \hat{y})) \leqq 0 \quad \text { for all }(u, v) \in X \times Y .
$$

Therefore, the theorem reduces to its special case where $X=Y$ and $f(x, y)$ is skew-symmetric. Accordingly, it suffices to see that there exists at least one point $\hat{x} \in X$ such that $f(s, \hat{x}) \leqq 0$ for all $s \in X$.

Suppose for the moment. that for any finite subset $F_{\lambda} \subset X(\lambda \in \Lambda)$, the set $N_{\lambda}=\left\{x \mid x \in X, f(s, x) \leqq 0\right.$ for all $\left.s \in F_{\lambda}\right\}$ is not empty. Then the family of closed subsets $\left\{N_{\lambda} \mid \lambda \in \Lambda\right\}$ admits finite intersection property and therefore $\bigcap_{\lambda \in \Lambda} N_{\lambda} \neq \varnothing$ because of the compactness of $X$. Thus every point $\hat{x} \in \bigcap_{\lambda \in \Lambda} N_{\lambda}$ is naturally a desired point. On the basis of the foregoing argument we have only to show that for any finite number of points $s_{1}, s_{2}, \cdots, s_{n} \in X$ there is at least one point $\hat{x} \in X$ with $f\left(s_{i}, \hat{x}\right) \leqq 0(i=1,2, \cdots, n)$.

We now proceed to the intrinsic portion of the proof. To begin with, for any fixed $s, x, y \in X$, let

$$
\alpha(t)=f(s,(1-t) x+t y), \quad 0 \leqq t \leqq 1 .
$$

Since $(1-t) x+t y \in X$, this function is well-defined by virtue of the convexity of $X$. By the assumed convexity of $f(s, x)$ we have

$$
\alpha(t) \leqq f(s, x)+t(f(s, y)-f(s, x)), \quad 0 \leqq t \leqq 1,
$$

and also $\alpha(0)=f(s, x)$. Hence it follows that

$$
(1 / t)(\alpha(t)-\alpha(0)) \leqq f(s, y)-f(s, x), \quad 0<t \leqq 1 ;
$$

Now put, for any finite number of points $s_{1}, s_{2}, \cdots, s_{n} \in X$,

$$
\begin{aligned}
& \theta_{i}(x)=\max \left(f\left(s_{i}, x\right), 0\right), \\
& \Phi(x)=\sum_{i=1}^{n} \theta_{i}(x)^{2} .
\end{aligned}
$$

$\Phi(x)$ is obviously continuous on the compact set $X$ so that it takes on a minimum at some point $\hat{x} \in X$. It will be shown that $\Phi(\hat{x})=0$. To 
this end, for any $y \in X$, let $\psi(t)=\Phi((1-t) \hat{x}+t y), 0 \leqq t \leqq 1$. As $\psi(0)$ $=\Phi(\hat{x})=\min _{x \in X} \Phi(x)$, it follows that

$$
\psi(t) \geqq \psi(0) \quad \text { for } 1 \geqq t \geqq 0 .
$$

Consider now the function of $n$ variables $\alpha_{1}, \alpha_{2}, \cdots, \alpha_{n}$,

$$
W\left(\alpha_{1}, \alpha_{2}, \cdots, \alpha_{n}\right)=\sum_{i=1}^{n}\left[\max \left(\alpha_{i}, 0\right)\right]^{2} .
$$

This function is convex and continuously differentiable: its derivatives are simply given by $\partial W / \partial \alpha_{i}=2 \max \left(\alpha_{i}, 0\right)$. Consequently,

$$
W\left(\xi_{1}, \xi_{2}, \cdots, \xi_{n}\right)-W\left(\eta_{1}, \eta_{2}, \cdots, \eta_{n}\right) \leqq \sum_{i=1}^{n}\left(\xi_{i}-\eta_{i}\right)\left[\partial W / \partial \alpha_{i}\right]_{\alpha_{i}=\xi_{i}},
$$

that is

$$
\begin{aligned}
W\left(\xi_{1}, \xi_{2}, \cdots, \xi_{n}\right)-W\left(\eta_{1}, \eta_{2}, \cdots\right. & \left., \eta_{n}\right) \\
& \leqq 2 \sum_{i=1}^{n}\left(\xi_{i}-\eta_{i}\right) \max \left(\xi_{i}, 0\right) .
\end{aligned}
$$

Now, if we put $\alpha_{i}(t)=f\left(s_{i},(1-t) \hat{x}+t y\right), 0 \leqq t \leqq 1$, we see $\psi(t)$ $=W\left(\alpha_{1}(t), \alpha_{2}(t), \cdots, \alpha_{n}(t)\right)$. Hence by (12),

$$
\psi(t)-\psi(0) \leqq 2 \sum_{i=1}^{n}\left(\alpha_{i}(t)-\alpha_{i}(0)\right) \max \left(\alpha_{i}(t), 0\right) .
$$

Therefore relations (8), (11) and (13) altogether yield

$$
\sum_{i=1}^{n}\left(f\left(s_{i}, y\right)-f\left(s_{i}, \hat{x}\right)\right) \max \left(\alpha_{i}(t), 0\right) \geqq 0, \quad 1 \geqq t>0 .
$$

By virtue of the fact that $\lim _{t \rightarrow 0} \max \left(\alpha_{i}(t), 0\right)=\max \left(\alpha_{i}(0), 0\right)=\theta_{i}(\hat{x})$, letting $t \rightarrow 0$ gives

$$
\sum_{i=1}^{n} \theta_{i}(\hat{x})\left(f\left(s_{i}, y\right)-f\left(s_{i}, \hat{x}\right)\right) \geqq 0,
$$

which shows

$$
\sum_{i=1}^{n} \theta_{i}(\hat{x}) f\left(s_{i}, y\right) \geqq \sum_{i=1}^{n} \theta_{i}(\hat{x})^{2}
$$

because $\theta_{i}(\hat{x}) f\left(s_{i}, \hat{x}\right)=\theta_{i}(\hat{x})^{2}$ in the light of (9). We have thereby proved that (16) is valid for any $y \in X$. In particular, for $y=s_{j}$, relation (16) is 


$$
\sum_{i=1}^{n} \theta_{i}(\hat{x}) f\left(s_{i}, s_{j}\right) \geqq \sum_{i=1}^{n} \theta_{i}(\hat{x})^{2}, \quad(j=1,2, \cdots, n) .
$$

Multiplying the $j$ th relation in $(17)$ by $\theta_{j}(\hat{x}) \geqq 0$ gives

$$
\sum_{i, j=1}^{n} f\left(s_{i}, s_{j}\right) \theta_{i}(\hat{x}) \theta_{j}(\hat{x}) \geqq \sum_{j=1}^{n} \theta_{j}(\hat{x}) \sum_{i=1}^{n} \theta_{i}(\hat{x})^{2} .
$$

Since $f(s, x)$ is skew-symmetric, the left-hand side of (18) vanishes so that $\Phi(\hat{x})=\sum_{i=1}^{n} \theta_{i}(\hat{x})^{2}=0$. This proves that $f\left(s_{i}, \hat{x}\right) \leqq \theta_{i}(\hat{x})=0$ $(i=1,2, \cdots, n)$, as asserted.

3. Some simplification for special cases. In the above proof a somewhat delicate argument was needed to meet the convexity of the payoff rather than its linearity. This portion of the proof will be dispensed with, if linearity is assumed. To be more specific, if

$f(s,(1-t) x+t y)=(1-t f(s, x)+t f(s, y)$ for $0 \leqq t \leqq 1, s, x, y \in X$, then the equality sign prevails in (7) as well as (8). Hence, on directly differentiating $\psi(t)$, we can evaluate its right-hand side derivative at $t=0$, and $D_{+} \psi(0)=2 \sum_{i=1}^{n} \theta_{i}(\hat{x})\left(f\left(s_{i}, y\right)-f\left(s_{i}, \hat{x}\right)\right)$. Also, in view of (11), we see $D_{+} \psi(0) \geqq 0$. Therefore these two relations yield (16).

Next some additional remarks will be made for two familiar special cases, J. Ville's minimax theorem regarding continuous games over the unit square and the corresponding theorem on matrix games. In these cases, namely, $\Phi(x)$ in (10), the function to be minimized, can be constructed in such a way that any point $x$ minimizing $\Phi(x)$ is nothing other than a saddle point for $f(s, x)$, whereas in general cases its construction is made in an approximative way on the basis of the finiteintersection-property argument.

(a) J. Ville's CASE. Let $H(\xi, \eta)$ be a continuous function for $\xi, \eta \in[0,1]$. The randomized payoff is given by

$$
f(F, G)=\int_{0}^{1} \int_{0}^{1} H(\xi, \eta) d F(\xi) d G(\eta)
$$

$F, G$ being probability distribution functions on $[0,1]$. The minimax theorem reduces to the existence of a pair $(\hat{F}, \hat{G})$ of probability distributions which fulfills $K\left(\left(\xi_{1}, \eta_{1}\right),(\hat{F}, \hat{G})\right) \leqq 0$ for $\xi_{1}, \eta_{1} \in[0,1]$, where

$$
K\left(\left(\xi_{1}, \eta_{1}\right),(F, G)\right)=\int_{0}^{1} H\left(\xi_{1}, \eta_{2}\right) d G\left(\eta_{2}\right)-\int_{0}^{1} H\left(\xi_{2}, \eta_{1}\right) d F\left(\xi_{2}\right) .
$$

The set $P$ of all probability distribution functions can be regarded as a $w^{*}$-compact convex subset in the conjugate space of the Banach 
space formed by all continuous functions on the unit square. Moreover, $K\left(\left(\xi_{1}, \eta_{1}\right),(F, G)\right)$ is $w^{*}$-continuous on $P \times P$ for each fixed pair $\left(\xi_{1}, \eta_{1}\right)$. The family of functions $K\left(\left(\xi_{1}, \eta_{1}\right),(F, G)\right)$ indexed by $(F, G)$ is uniformly equi-continuous. Thus, $K\left(\left(\xi_{1}, \eta_{1}\right),(F, G)\right)$ is simultaneously continuous with respect to $\xi_{1}, \eta_{1}, F, G$. Since its domain of definition, $[0,1] \times[0,1] \times P \times P$ is compact, this function is even uniformly continuous. Based on this result we see that

$$
\theta\left(\left(\xi_{1}, \eta_{1}\right),(F, G)\right)=\max \left(K\left(\left(\xi_{1}, \eta_{1}\right),(F, G)\right), 0\right)
$$

is integrable on the unit square in the Riemann's sense for each fixed $(F, G)$, and that

$$
\Phi((F, G))=\int_{0}^{1} \int_{0}^{1} \theta\left(\left(\xi_{1}, \eta_{1}\right),(F, G)\right)^{2} d \xi_{1} d \eta_{1}
$$

is continuous on $P \times P$. The direct application of the method given in $\$ 2$, with the simplification described above on mind, will show that if $(\hat{F}, \hat{G})$ minimizes $\Phi((F, G)), \Phi((\hat{F}, \hat{G}))$ must vanish, which gives $\theta\left(\left(\xi_{1}, \eta_{1}\right),(\hat{F}, \hat{G})\right)=0$ for $\xi_{1}, \eta_{1} \in[0,1]$ because of the continuity of $\theta$.

(b) CASE of matrix Games. Every game with a nonskew-symmetric payoff matrix reduces to a game with a skew-symmetric payoff matrix in one of several well-known fashions. For any skew-symmetric payoff matrix $A=\left(a_{i j}\right)$, the corresponding function to be minimized is

$$
\Phi(x)=\sum_{i=1}^{n} \theta_{i}(x)^{2},
$$

where $\theta_{i}(x)=\max \left(\sum_{j=1}^{n} a_{i j} x_{j}, 0\right)$.

4. Duality in linear programming. In this section rearrangement will be made of the method of proof to derive duality in linear programming. To assure the existence of a point minimizing a certain function corresponding to (10) use will be made of the closedness of the convex cones generated by a finite number of points in contrast with the discussion in the foregoing sections where the compactness of the spaces is available.

In the sequel $x \geqq y$ will be used to designate the ordinary semiorder based on coordinatewise comparison among vectors. A prime implies transposition. To avoid the complication of symbols the following convention is made: Subscripts in Roman letters refer to coordinates of points whereas those in Greek letters refer to members of a sequence of points.

Lemma 1. Let $\Phi(x)$ be defined for non-negative $x$ 's of a Euclidean $n$ 
space $R^{n}$, and continuously differentiable there. If $\Phi(\hat{x})=\min _{x \geqq 0} \Phi(x)$, then we have

$$
\begin{aligned}
\frac{\partial}{\partial x_{i}} \Phi(\hat{x}) & \geqq 0 \quad(i=1,2, \cdots, n) \\
\sum_{i=1}^{n} \hat{x}_{i} \frac{\partial}{\partial x_{i}} \Phi(\hat{x}) & =0 .
\end{aligned}
$$

Proof. In accordance with the argument in $\$ 2$, let for any fixed $y \geqq 0, \quad y \in R^{n}, \quad \psi(t)=\Phi((1-t) \hat{x}+t y)$. As before, from $\psi(t) \geqq \psi(0)$ $(1 \geqq t \geqq 0)$ follows

$$
0 \leqq D_{+} \psi(0)=\sum_{i=1}^{n}\left(y_{i}-\hat{x}_{i}\right) \frac{\partial}{\partial x_{i}} \Phi(\hat{x})
$$

for any $y \geqq 0, y \in R^{n}$, from which (21) and (22) are easily seen.

Lemma 2. Let $A=\left(a_{i j}\right)$ be an $m \times n$ matrix and $x \in R^{n}, b, b_{\nu} \in R^{m}$ $(\nu=1,2, \cdots)$. If $X_{\nu}=\left\{x \mid x \geqq 0, A x \leqq b_{\nu}\right\} \neq \varnothing,(\nu=1,2, \cdots)$, and $\lim _{\nu \rightarrow \infty} b_{\nu}=b$, then $X=\{x \mid x \geqq 0, A x \leqq b\} \neq \varnothing$.

Proof. Since $X_{\nu} \neq \varnothing$, we have $b_{\nu}=A x_{\nu}+u_{\nu}$ for some $x_{\nu} \geqq 0, u_{\nu} \geqq 0$. Let $I$ be the $m \times m$ identity matrix. Then every $b_{\nu}$ belongs to the convex cone generated by the column vectors of the matrix $(A, I)$. As this cone is closed, it also contains $b$, which implies $X \neq \varnothing$.

Lemma 3. For an $m \times n$ matrix $A=\left(a_{i j}\right)$ and a point $b \in R^{m}$, let $\Phi(x)=\sum_{i=1}^{m} \theta_{i}(x)^{2}$, where $\theta_{i}(x)=\max \left(\sum_{j=1}^{n} a_{i j} x_{j}+b_{i}, 0\right), x \in R^{n}$. The function $\Phi(x)$ takes on a minimum over all $x \geqq 0$.

Proof. Let $\inf _{x \geqq 0} \Phi(x)=\delta \geqq 0$. Take a sequence $\left\{x_{\nu}\right\}$ such that $\lim _{\nu \rightarrow \infty} \Phi\left(x_{\nu}\right)=\delta$. As $\left\{\theta_{i}\left(x_{\nu}\right)\right\}$ is bounded for each $i$, it may be assumed that $\lim _{\nu \rightarrow \infty} \theta_{i}\left(x_{\nu}\right)=\delta_{i}$ exists $(i=1,2, \cdots, m)$. Let $\theta\left(x_{\nu}\right)$ and $d$ be vectors whose $i$ th coordinates are $\theta_{i}\left(x_{\nu}\right)$ and $\delta_{i}$ respectively. The set $X_{\nu}=\left\{x \mid x \geqq 0, A x \leqq \theta\left(x_{\nu}\right)-b\right\}$ is nonempty for each $\nu$, as $X_{\nu} \ni x_{\nu}$. Moreover $\lim _{\nu \rightarrow \infty}\left(\theta\left(x_{v}\right)-b\right)=d-b$. By Lemma 2, we therefore have $X=\left\{x \mid x \geqq 0, \theta_{i}(x) \leqq \delta_{i}(i=1,2, \cdots, m)\right\}=\{x \mid x \geqq 0, A x \leqq d-b\}$ $\neq \varnothing$. As obviously $\sum_{i=1}^{m} \delta_{t}^{2}=\delta$, we see that if $\hat{x} \in X$, then $\delta \leqq \Phi(\hat{x})$ $\leqq \sum_{i=1}^{m} \delta_{i}^{2}=\delta$ Hence, $\Phi(\hat{x})=\delta$, which was to be shown.

Duality theorem. Let $A=\left(a_{i j}\right)$ be an $m \times n$ matrix and $x, b \in R^{m}$, $y, c \in R^{n}$, where $R^{m}$ and $R^{n}$ are Euclidean $m$ and $n$-spaces respectively. If $X=\left\{x \mid x \geqq 0, x^{\prime} A \geqq c^{\prime}\right\}$ as well as $Y=\{y \mid y \geqq 0, A y \leqq b\}$ is nonempty, then we have

(i) $b^{\prime} x \geqq c^{\prime} y$ for all $x \in X, y \in Y$.

(ii) $b^{\prime} \hat{x}=c^{\prime} \hat{y}$ for some $\hat{x} \in X, \hat{y} \in Y$. 
Proof. By virtue of (i) which is easily seen, it suffices to show that $b^{\prime} \hat{x} \leqq c^{\prime} \hat{y}$ for some $\hat{x} \in X, \hat{y} \in Y$. To this end, let us define

$$
\sigma_{i}(y)=\max \left(\sum_{j=1}^{n} a_{i j} y_{j}-b_{i}, 0\right), \quad(i=1,2, \cdots, m),
$$

$$
\tau_{j}(x)=\max \left(c_{j}-\sum_{i=1}^{m} a_{i j} x_{i}, 0\right), \quad(j=1,2, \cdots, n)
$$

$$
\begin{aligned}
& \rho(x, y)=\max \left(\sum_{i=1}^{m} b_{i} x_{i}-\sum_{j=1}^{n} c_{j} y_{j}, 0\right) \\
& \Phi(x, y)=\sum_{i=1}^{m} \sigma_{i}(y)^{2}+\sum_{j=1}^{n} \tau_{j}(x)^{2}+\rho(x, y)^{2}
\end{aligned}
$$

for $x \in R^{m}, y \in R^{n}$. This function (26) is nothing else than $\Phi$ as defined in Lemma 3 for the $(m+n+1) \times(m+n)$ matrix

$$
\left(\begin{array}{cc}
0 & A \\
-A^{\prime} & 0 \\
b^{\prime} & -c^{\prime}
\end{array}\right)
$$

Therefore, by this lemma, there is a pair $(\hat{x}, \hat{y}) \geqq 0$ that minimizes $\Phi(x, y)$ over all $x \geqq 0, y \geqq 0$. It will be shown that $\Phi(\hat{x}, \hat{y})=0$ whenever $X \neq \varnothing, Y \neq \varnothing$. Since $\Phi(x, y)$ is continuously differentiable, Lemma 1 can be applied. Hence, on differentiating $\Phi(x, y)$, we have

$$
\frac{\partial}{\partial x_{i}} \Phi(\hat{x}, \hat{y})=-2 \sum_{j=1}^{n} a_{i j} \tau_{j}(\hat{x})+2 \rho(\hat{x}, \hat{y}) b_{i} \geqq 0,(i=1,2, \cdots, m),
$$

$$
\frac{\partial}{\partial y_{i}} \Phi(\hat{x}, \hat{y})=2 \sum_{i=1}^{m} a_{i j} \sigma_{i}(\hat{y})-2 \rho(\hat{x}, \hat{y}) c_{j} \geqq 0, \quad(j=1,2, \cdots, n),
$$

$$
-\sum_{j=1}^{n} \tau_{j}(\hat{x}) \sum_{i=1}^{m} a_{i j} \hat{x}_{i}+\sum_{i=1}^{m} \sigma_{i}(\hat{y}) \sum_{j=1}^{n} a_{i j} \hat{y}_{j}
$$

$$
+\rho(\hat{x}, \hat{y})\left(\sum_{i=1}^{m} b_{i} \hat{x}_{i}-\sum_{j=1}^{n} c_{j} \hat{y}_{j}\right)=0 .
$$

Now, in view of (24), we see

$$
\tau_{j}(\hat{x})^{2}=\tau_{j}(\hat{x})\left(c_{j}-\sum_{i=1}^{m} a_{i j} \hat{x}_{i}\right)=c_{j} \tau_{j}(\hat{x})-\tau_{j}(\hat{x}) \sum_{i=1}^{m} a_{i j} \hat{x}_{i},
$$

and similarly 


$$
\begin{aligned}
\sigma_{i}(\hat{y})^{2} & =\sigma_{i}(\hat{y}) \sum_{j=1}^{n} a_{i j} \hat{y}_{j}-b_{i} \sigma_{i}(\hat{y}), \\
\rho(\hat{x}, \hat{y})^{2} & =\rho(\hat{x}, \hat{y})\left(\sum_{i=1}^{m} b_{i} \hat{x}_{i}-\sum_{j=1}^{n} c_{j} \hat{y}_{j}\right) .
\end{aligned}
$$

Using these relations, we can rewrite (29) as follows:

$$
\begin{aligned}
\Phi(\hat{x}, \hat{y}) & =\sum_{i=1}^{m} \sigma_{i}(\hat{y})^{2}+\sum_{j=1}^{n} \tau_{j}(\hat{x})^{2}+\rho(\hat{x}, \hat{y})^{2} \\
& =\sum_{j=1}^{n} c_{j} \tau_{j}(\hat{x})-\sum_{i=1}^{m} b_{i} \sigma_{i}(\hat{y}) .
\end{aligned}
$$

We now prove that $\Phi(\hat{x}, \hat{y})=0$ whenever $X$ and $Y$ are nonempty. To this end, let $\gamma=\inf \left(b^{\prime} p-c^{\prime} q\right) \geqq 0$ for $p \in X, q \in Y$. For any $\epsilon>0$ take $p \in X, q \in Y$ such that $\gamma+\epsilon>b^{\prime} p-c^{\prime} q$. Designate by $\sigma(\hat{y}), \tau(\hat{x})$ the vectors whose components are $\sigma_{i}(\hat{y})$ and $\tau_{j}(\hat{x})$ respectively. Then, in the light of (27), (28), a simple computation yields

$$
\frac{\sigma(\hat{y})+p}{1+\rho(\hat{x}, \hat{y})} \in X, \quad \frac{\tau(\hat{x})+q}{1+\rho(\hat{x}, \hat{y})} \in Y .
$$

Hence, by (i),

or,

$$
b^{\prime} \frac{\sigma(\hat{y})+p}{1+\rho(\hat{x}, \hat{y})}-c^{\prime} \frac{\tau(\hat{x})+q}{1+\rho(\hat{x}, \hat{y})} \geqq \gamma>b^{\prime} p-c^{\prime} q-\epsilon,
$$

$$
b^{\prime} \sigma(\hat{y})-c^{\prime} \tau(\hat{x})+b^{\prime} p-c^{\prime} q>(1+\rho(\hat{x}, \hat{y}))\left(b^{\prime} p-c^{\prime} q-\epsilon\right),
$$

from which follows $b^{\prime} \sigma(\hat{y})-c^{\prime} \tau(\hat{x})>-\epsilon(1+\rho(\hat{x}, \hat{y}))$. Thus the arbitrariness of $\epsilon$ implies $b^{\prime} \sigma(\hat{y})-c^{\prime} \tau(\hat{x}) \geqq 0$. This result, combined with relation (30), means $0 \leqq \Phi(\hat{x}, \hat{y})=c^{\prime} \tau(\hat{x})-b^{\prime} \sigma(\hat{y}) \leqq 0$, that is $\Phi(\hat{x}, \hat{y})=0$. Accordingly, $b^{\prime} \hat{x} \leqq c^{\prime} \hat{y}$ because $b^{\prime} \hat{x}-c^{\prime} \hat{y} \leqq \rho(\hat{x}, \hat{y})=0$. Moreover $\hat{x} \in X$, $\hat{y} \in Y$, since $c-A^{\prime} \hat{x} \leqq \tau(\hat{x})=0$ and $A \hat{y}-b \leqq \sigma(\hat{y})=0$. This completes the proof.

\section{REFERENCES}

1. G. W. Brown and J. von Neumann, Solutions of games by differential equations, Annals of Mathematics Studies, no. 24.

2. D. Gale, H. W. Kuhn and A. W. Tucker, On symmetric games, ibid.

3. H. Nikaidô, On von Neumann's minimax theorem, Pacific J. Math. vol. 4, no. 1 .

4. H. Nikaidô and K. Isoda, Note on noncooperative convex games, Pacific J. Math., vol. 5 , supplement.

5. H. Nikaido, Minimax Têri no Shomei ni tsuite, Sugaku. vol. 10, no. 1.

6. M. Sion, On general minimax theorems, Pacific J. Math. vol. 8, no. 1 (1958).

Osaka University, Osaka, Japan 